\title{
A Randomized, Phase 2 Study of 24-h Efficacy and Tolerability of Netarsudil in Ocular Hypertension and Open-Angle Glaucoma
}

\author{
James H. Peace · Hayley J. McKee · Casey C. Kopczynski
}

Received: October 22, 2020 / Accepted: November 11, 2020 / Published online: November 26, 2020

(C) The Author(s) 2020

\begin{abstract}
Introduction: Pharmacotherapy to lower intraocular pressure (IOP) is a mainstay of treatment aimed at delaying progression of visual field loss in ocular hypertension (OHT) and open-angle glaucoma (OAG), but some topical treatments are less effective in controlling IOP at night. Peak IOP may be related to glaucoma progression and can occur outside office hours. A phase 2 study was conducted to evaluate the IOP-lowering efficacy of netarsudil across the diurnal and nocturnal periods.

Methods: This was a randomized, doublemasked, single-center, vehicle-controlled, 9-day study. After washout of any prior ocular hypotensive agents, 12 patients with OHT or OAG underwent baseline IOP assessment at 15:00, 18:00, 21:00, 00:00, 03:00, 06:00, 09:00, and $12: 00 \mathrm{~h}$ on day 1 /day 2 . Participants were then randomized in a $2: 1$ ratio to netarsudil ophthalmic solution $0.02 \%(n=8)$ or vehicle $(n=4)$ for 7 days of self-administered dosing each evening. IOP was assessed at the same time points on day 8/day 9. All measurements were
\end{abstract}

J. H. Peace $(\square)$

United Medical Research Institute, Inglewood, CA,

USA

e-mail: drpeace@drjamespeace.com

H. J. McKee · C. C. Kopczynski

Aerie Pharmaceuticals, Inc., Durham, NC, USA conducted with a Perkins tonometer in habitual positions by day (seated) and at night (supine). Results: Baseline mean 24-h IOP was $22.4 \mathrm{mmHg}$ in the netarsudil group and $22.9 \mathrm{mmHg}$ in the vehicle group. Netarsudil was associated with a reduction in mean nocturnal IOP (measurements at 21:00, 00:00, 03:00, 06:00 h) of $3.5 \mathrm{mmHg}$, which was significant relative to baseline nocturnal IOP $(P<0.001)$ and the reduction in the vehicle group $(0.4 \mathrm{mmHg} ; P<0.001$ vs. netarsudil). Reduction in mean diurnal IOP with netarsudil (3.5 $\mathrm{mmHg}$ ) was the same as the nocturnal reduction and statistically significant versus baseline $(P<0.001)$ and the vehicle group $(0.9 \mathrm{mmHg} ; P<0.01)$. The magnitude of IOP reductions with netarsudil was consistent at each time point assessed over the 24 -h period. No adverse events were reported.

Conclusion: Netarsudil exhibited consistent IOP-lowering efficacy over a 24 -h period in this short-term study.

Trial Registration: Clinicaltrials.gov identifier: NCT02874846.

\section{PLAIN LANGUAGE SUMMARY}

When pressure inside the eye (called intraocular pressure [IOP]) builds up, a patient may develop a condition known as glaucoma, in which damage to the optic nerve and possibly 
irreversible vision loss occur. Glaucoma can be preceded in some patients by a condition called ocular hypertension (OHT). Patients with OHT and the most common type of glaucoma (openangle glaucoma [OAG]) should be treated to lower their IOP and decrease the risk for progressive visual loss. Several studies that have evaluated 24-h IOP control have indicated that some eye drops lower IOP less effectively at night than during the day. A pilot study was conducted in 12 patients with OHT or OAG to evaluate netarsudil's IOP lowering effect during the day and at night. After a week of treatment with netarsudil or a similar eye drop that did not contain the active drug, patients who took netarsudil experienced the same decrease in IOP at night as during the day. IOP was statistically lower with netarsudil than with the drug-free comparator both during the day and at night. Although this was a small study in 12 patients, the results are of interest because they suggest that netarsudil might consistently reduce IOP over a 24 -h period.

Keywords: 24-h; Clinical study; Intraocular pressure; Netarsudil; Nocturnal; Ocular hypertension; Open-angle glaucoma

\section{Key Summary Points}

Why carry out this study?

Topical agents to lower intraocular pressure (IOP) have demonstrated efficacy in clinical trials, but IOP may spike at time points during the night that are outside office hours.

Trials assessing IOP control over a $24-\mathrm{h}$ period have shown that some classes of IOP-lowering medication have reduced efficacy or no efficacy at night.

The aim was to evaluate netarsudil efficacy over a 24-h period in patients with ocular hypertension or open-angle glaucoma.

\section{What was learned from the study?}

Netarsudil exhibited consistent IOP-

lowering efficacy over a 24 -h period, with

the same reduction from baseline in mean

nocturnal IOP $(3.5 \mathrm{mmHg})$ and mean

diurnal IOP (3.5 mmHg).

Results of this short-term pilot study

$(N=12)$ suggest that further research on

the 24-h efficacy of netarsudil is

warranted.

\section{DIGITAL FEATURES}

This article is published with digital features, including a summary slide and plain language summary, to facilitate understanding of the article. To view digital features for this article go to https://doi.org/10.6084/m9.figshare. 13214831

\section{INTRODUCTION}

Glaucoma is a common condition, with a prevalence of $3.5 \%$ in adults aged $40-80$ years and the potential to lead to serious vision loss [1]. The pathophysiology of glaucoma is complex, with multiple vascular, hemodynamic, and mechanical risk factors believed to contribute to optic nerve damage and visual field loss [2]. Given a recent estimate of 4 million current glaucoma patients with moderate to severe visual impairment globally and another 3 million cases of blindness, halting or slowing glaucoma disease progression is imperative [3]. Elevated intraocular pressure (IOP) is a major risk factor for visual field loss in patients with ocular hypertension (OHT) or open-angle glaucoma (OAG), and interventions to reduce IOP have been shown to delay disease progression in these populations [4-9]. Large diurnal fluctuations in IOP are an independent risk factor for progression in patients with glaucoma [10]. Peak IOP is related to glaucoma progression, and previous research has shown that IOP peaks 
in glaucoma patients frequently occur at times of the day that are outside of office hours [11].

Abnormally low ocular perfusion pressure, measured as the difference between systemic arterial pressure and IOP, may compromise blood supply to the optic nerve head [12]. Supporting a pathophysiologic role for inadequate ocular perfusion pressure, nocturnal dipping in systemic blood pressure and associated fluctuations in ocular perfusion pressure might increase the risk of glaucoma progression $[13,14]$

One goal of ocular hypotensive treatment therefore should be the maintenance of reduced IOP throughout a 24 -h period, i.e., during the night as well as during the day. Netarsudil, a Rho kinase (ROCK) inhibitor, has been previously shown to lower IOP in clinical trials that evaluated efficacy at conventional, diurnal time points (08:00, 10:00, and 16:00 h) [15]. The purpose of this study was to assess the nocturnal ocular hypotensive efficacy of netarsudil by collecting data over a full 24 -h period, based on the hypothesis that netarsudil's mechanism of action would produce similar IOP-lowering efficacy both during the night and during the day.

\section{METHODS}

\section{Participants}

To be included, subjects were required to be adults aged $\geq 18$ years with a diagnosis of OHT or OAG in both eyes (OAG in one eye and OHT in the fellow eye was acceptable), to have unmedicated (post-washout) IOP $>17 \mathrm{mmHg}$ in one or both eyes and $<30 \mathrm{mmHg}$ in both eyes at the qualification visit (i.e., 2-7 days prior to day 1), and to have corrected visual acuity in each eye of +1.0 logMAR (equivalent to 20/200) or better using the Early Treatment Diabetic Retinopathy Study (ETDRS) chart. The required minimum duration of washout before the qualification visit was determined by the medication class used: 4 weeks for prostaglandin analogues and beta-adrenoceptor antagonists; 2 weeks for adrenergic agonists (including alpha agonists); or 5 days for muscarinic agonists and carbonic anhydrase inhibitors (topical or oral formulations).

The key ocular exclusion criteria were: glaucoma with pseudoexfoliation or a pigment dispersion component, history of angle closure, or narrow angles; previous laser peripheral iridotomy; IOP $\geq 30 \mathrm{mmHg}$ at the qualification visit, use of $>2$ ocular hypotensive medications within 30 days of screening (fixed-dose combinations counted as two medications); previous glaucoma intraocular surgery or glaucoma laser procedures in the study eye(s); refractive surgery in study eye(s) (radial keratotomy, photorefractive keratotomy, laser-assisted in situ keratomileusis, etc.); central corneal thickness $>620 \mu \mathrm{m}$ at screening. Systemic exclusion criteria included: a sleep disorder or an irregular sleep schedule; changes to systemic medication that could have a substantial effect on IOP and/or visual field within 30 days prior to screening, or anticipated during the study; and pregnancy in women of childbearing potential.

\section{Design}

This was a double-masked, randomized, singlecenter, vehicle-controlled, interventional clinical study (Clinicaltrials.gov identifier: NCT02874846). Following screening and qualification visits, participants reported to the study center beginning on the afternoon of day 1 and underwent IOP evaluations every $3 \mathrm{~h}$ beginning at $15: 00 \mathrm{~h}$ and continuing through $12: 00 \mathrm{~h}$ on day 2 (Fig. 1). At the end of the visit on day 2, participants were randomized 2:1 to netarsudil ophthalmic solution $0.02 \%$ or vehicle, then checked out of the study center with instructions to instill the assigned study drug as 1 drop in each eye, once daily between 20:00 and 22:00 h. On day 8, subjects returned to the study center for IOP evaluations at multiple time points on the same schedule as on day $1 /$ day 2 , starting at 15:00 h on day 8 and ending at 12:00 h on day 9. The visit included administration of the final evening dose of study drug on day 8. After completion of the visit procedures on day 9, participants were discharged from the study center and exited the study. 

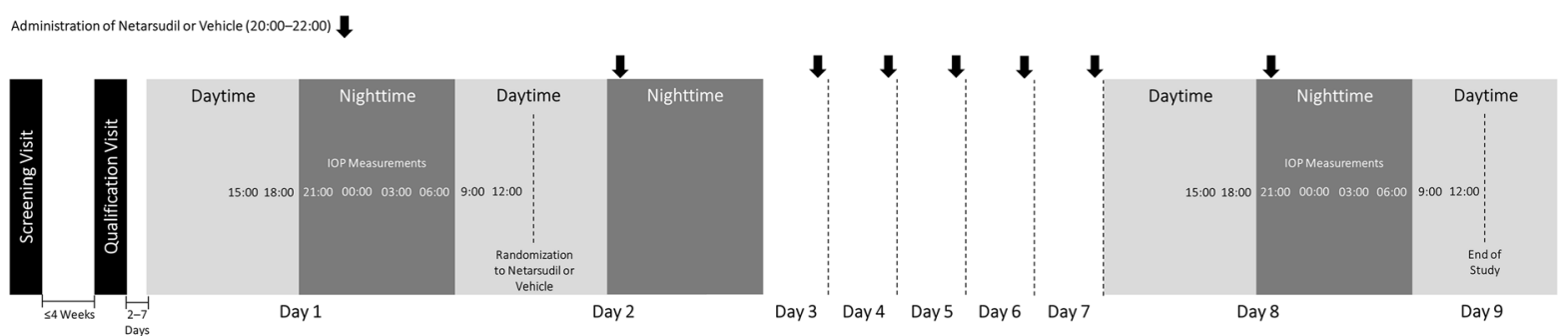

Fig. 1 Flow diagram of study design. IOP Intraocular pressure

The randomization schedule was prepared by the sponsor representative to match the planned randomization ratio. The study center was provided a predefined order sequence (i.e., kit number) for the dispensation of the study drug to patients.

The investigator, subjects, sponsor, sponsor representative, and clinical monitors were not allowed to know the treatment assigned to each subject. Thus, the clinical assessments should not have been influenced by the knowledge of an individual subject's specific study drug assignment. Active and vehicle study medications were packaged in identical bottles with identical labels, with the exception of a unique kit number identifier on the label. Additionally, there were no obvious differences in appearance (e.g., color or viscosity) between the netarsudil and vehicle solutions. Use of lacrimal occlusion was not required in the patient instructions for study drug instillation.

\section{Assessments}

At the screening visit, patients provided informed consent and were assessed for medical/ophthalmic history (including any current symptoms/adverse events), concomitant medications, eligibility for participation (including a pregnancy test in women of childbearing potential), and vital signs (heart rate and blood pressure). Evaluation of both eyes included visual acuity using an ETDRS chart, IOP, corneal thickness by pachymetry, biomicroscopy of the anterior segment, including evaluation of corneal epithelium, bulbar and lower conjunctiva, and lens, dilated fundus examination, and gonioscopy and visual field testing. Gonioscopy and visual field testing could have been performed at this visit or up to 3 months prior to randomization. Patients were instructed on the required washout periods for ocular hypotensive medication.

A qualification visit took place between 12:00 and 16:00 $\mathrm{h}$ up to 4 weeks after screening, depending on the required washout. Patient medical history and use of concomitant medications were updated, including confirmation of washout. Symptoms/adverse events, vital signs, and study eligibility were again assessed. Ocular assessments of both eyes consisted of visual acuity by ETDRS chart, IOP, and biomicroscopy.

On day 1, which was 2-7 days after the qualification visit, patients were assessed at 15:00 $\mathrm{h}$ for any changes to medical history and medications used, vital signs, adverse events, visual acuity by ETDRS chart, biomicroscopic features, and IOP. Additional IOP measurements of both eyes were taken every $3 \mathrm{~h}$, at 18:00 and 21:00 $\mathrm{h}$ on day 1 , and at 00:00, 03:00, 06:00, 09:00, and 12:00 $\mathrm{h}$ on day 2 . After the last IOP assessment, patients were queried again about adverse events, assigned to their study drug, and discharged. Subjects returned for a visit on day 8/day 9 to undergo the same assessments at the same times as on day 1 /day 2 .

IOP was measured at each time point by study staff masked to treatment using a Perkins tonometer. Assessments of IOP were performed in habitual positions over the 24-h period, namely, in a seated position during the day $(09: 00,12: 00,15: 00,18: 00 \mathrm{~h})$ and in a supine position at night (21:00, 00:00, 03:00, and 06:00 h). At each time point, two consecutive IOP measurements of each eye were obtained. If the two measurements differed by more than $2 \mathrm{mmHg}$, the investigator was instructed to 
obtain a third measurement. IOP for each time point was analyzed as the mean of two measurements or as the median of three measurements.

Adverse events were elicited from participants at study visits, and treatment emergent adverse events were recorded after day 2 (first PM dosing); those recorded at baseline were included in the patient's medical history.

The study eye was defined as the eye that met both the IOP and the ocular history inclusion/exclusion requirements. If both eyes qualified, the study eye with the higher nocturnal mean IOP at day 1 /day 2 was designated as the study eye. If both eyes had the same mean nocturnal IOP at day 1 /day 2 , the right eye was designated as the study eye.

\section{Study Endpoints}

The primary efficacy endpoint was mean change from baseline in mean nocturnal IOP (defined as the mean of the four nocturnal time points: 21:00, 00:00, 03:00, and 06:00 h) at day 8/day 9. The secondary efficacy endpoints as prespecified in the statistical analysis plan were:

- Mean change from baseline in mean diurnal IOP (defined as the mean of the four diurnal time points: 09:00, 12:00, 15:00, 18:00 h) at day 8 /day 9 ;

- Mean change from baseline in mean 24-h IOP (defined as the mean of all eight time points) at day 8/day 9;

- Mean change from baseline IOP at each posttreatment time point at day 8/day 9;

- Mean IOP at each post-treatment time point at day 8/day 9;

- Difference between mean change from baseline nocturnal IOP and mean change from baseline diurnal IOP within the netarsudil treatment group.

Assessment of systemic and ocular safety was a secondary objective of the study.

\section{Statistical Analyses}

Sample size was determined based on the following calculations: assuming a common standard deviation (SD) of $3.0 \mathrm{mmHg}$ within the active treatment group at each time point, a $60 \%$ correlation among time points (yielding an SD of 2.51 for the mean change from baseline in the four nocturnal time points), and a two-sided alpha of 0.10, a sample size of eight subjects in the active treatment group would yield $80 \%$ power to demonstrate that the mean change from baseline in the mean nocturnal IOP (IOP day 8/day 9 minus IOP baseline) is significantly less than zero, assuming a mean change from baseline of $2.5 \mathrm{mmHg}$.

Assuming a common SD of 3.0 and $1.5 \mathrm{mmHg}$ within the active and vehicle groups, respectively, at each time point, a 60 and $20 \%$ correlation among time points for the active and vehicle groups, respectively (yielding SD of 2.51 and 1.0 for the mean change from baseline in the four nocturnal time points for the active and vehicle groups, respectively) and a twosided alpha of 0.10 , a sample size of eight patients in the active group and four patients in the vehicle group would yield $80 \%$ power to detect a difference between the active and vehicle groups for the mean change from baseline in the mean nocturnal IOP, assuming a mean difference of $2.75 \mathrm{mmHg}$.

The intent to treat (ITT) population served as the primary efficacy population. All randomized subjects who received at least one dose of study drug were included in the ITT population. Subjects in the ITT population were analyzed in accordance with their assigned randomized treatment, even if the actual treatment the subject received was different from the planned treatment. All subjects who received at least one dose of study drug were to be included in the safety population.

The mean change from baseline in mean nocturnal IOP within each treatment group was tested using one-sample $t$ tests and 90\% $t$-distribution confidence intervals around the mean change. Two-sample $t$ tests and 90\% $t$-distribution confidence intervals around the difference in the mean nocturnal IOP between the two treatments (netarsudil-vehicle) were employed, using the Satterthwaite approximation for unequal variance, as the primary statistical comparisons between netarsudil and vehicle. 


\section{Ethical Conduct}

The study site underwent a site qualification visit and was approved to participate in the study by the administrating institutional review board (IRB; Schulman Institutional Review Board, IRB\# 201604624). This study was conducted in accordance with IRB regulations. Additionally, ethical conduct of the study was ensured by adhering to the following: US Code of Federal Regulations, Title 21; International Conference on Harmonization-Consolidated Good Clinical Practices Guideline (E6); standard operating procedures of the sponsor and any vendors participating in the conduct of the study; and the ethical principles that have their origin in the Declaration of Helsinki. An approved informed consent procedure had to take place before any study-specific procedures were initiated.

\section{RESULTS}

\section{Patient Disposition and Characteristics}

Of the 12 patients randomized, $100 \%$ received their assigned study medication and therefore comprised the ITT population. All patients completed the study, and no protocol deviations were reported (Table 1).

Women and men were equally distributed in each treatment group, there were more patients aged $\geq 65$ years $\quad(66.7 \%) \quad$ than $<65$ years $(33.3 \%)$, and a higher percentage of subjects were black or African American (75.0\%) than any other race $(25.0 \%)$ (Table 2$)$. No patient had been previously treated with prostaglandins for their OAG or OHT, and two subjects were treatment-naïve upon screening. Of the ten patients who had been on prior ocular hypotensive therapy, the mean time on the most recent therapy was 62.1 weeks. Of the patients enrolled in the study, 92\% had OAG. Mean baseline (day 1/day 2) values for 24-h IOP, nocturnal IOP, and diurnal IOP, respectively (in $\mathrm{mmHg}$ ), in the study eye were $22.4,22.8$, and 22.0 in the netarsudil group and 22.9, 23.6, and 22.3 in the vehicle group. Values for cup to disc ratio and visual field indicated a population with early glaucoma.

\section{Efficacy}

Netarsudil met the primary efficacy endpoint, with a significant mean reduction from baseline in study eye mean nocturnal IOP $(3.5 \mathrm{mmHg}$; $P<0.001$ ) at day $8 /$ day 9 (Fig. 2a). Mean reduction from baseline in study eye mean

Table 1 Patient disposition

\begin{tabular}{llll}
\hline Patient disposition & Netarsudil treatment arm & Vehicle treatment arm & Total \\
\hline Patients randomized & 8 & 4 & 12 \\
Analysis populations & $8(100.0)$ & $4(100.0)$ & $12(100.0)$ \\
Safety & $8(100.0)$ & $4(100.0)$ & $12(100.0)$ \\
Intent-to-treat & $8(100.0)$ & & \\
Study completion & 0 & $4(100.0)$ & $12(100.0)$ \\
Completed & 0 & 0 & 0 \\
Discontinued & 0 & 0 & 0 \\
Missing & 0 & 0 & 0 \\
Protocol deviations & & & \\
\hline
\end{tabular}

Values are presented as the number of patients with the percentage given in parentheses 
Table 2 Patient baseline demographics and clinical characteristics

\begin{tabular}{|c|c|c|c|}
\hline $\begin{array}{l}\text { Baseline demographics and clinical } \\
\text { characteristics }\end{array}$ & $\begin{array}{l}\text { Netarsudil treatment arm } \\
(n=8)\end{array}$ & $\begin{array}{l}\text { Vehicle treatment arm } \\
(n=4)\end{array}$ & Total $(N=12)$ \\
\hline \multicolumn{4}{|l|}{ Age (years) } \\
\hline Mean \pm SD & $64.4 \pm 10.23$ & $64.5 \pm 5.07$ & $64.4 \pm 8.58$ \\
\hline Range & $47-75$ & $57-68$ & $47-75$ \\
\hline$<65$ & $3(37.5 \%)$ & $1(25.0 \%)$ & $4(33.3)$ \\
\hline$\geq 65$ & $5(62.5 \%)$ & $3(75.0)$ & $8(66.7)$ \\
\hline \multicolumn{4}{|l|}{ Gender } \\
\hline Female & $4(50.0 \%)$ & $2(50.0 \%)$ & $6(50.0)$ \\
\hline Male & $4(50.0 \%)$ & $2(50.0 \%)$ & $6(50.0)$ \\
\hline \multicolumn{4}{|l|}{ Ethnicity } \\
\hline Hispanic or Latino & $1(12.5 \%)$ & $1(25.0 \%)$ & $2(16.7)$ \\
\hline Not Hispanic or Latino & $7(87.5 \%)$ & $3(75.0 \%)$ & $10(83.3)$ \\
\hline \multicolumn{4}{|l|}{ Race } \\
\hline Asian & 0 & 0 & 0 \\
\hline Black/African American & $6(75.0 \%)$ & $3(75.0 \%)$ & $9(75.0)$ \\
\hline Native American & 0 & 0 & 0 \\
\hline White & $2(25.0 \%)$ & $1(25.0 \%)$ & $3(25.0)$ \\
\hline Multiple & 0 & 0 & 0 \\
\hline Other & 0 & 0 & 0 \\
\hline \multicolumn{4}{|l|}{ Iris color, study eye } \\
\hline Brown/black & $8(100.0 \%)$ & $4(100.0 \%)$ & $12(100.0)$ \\
\hline Blue/gray/green, hazel, or other & 0 & 0 & 0 \\
\hline \multicolumn{4}{|l|}{ Study eye diagnosis, } \\
\hline Ocular hypertension & 0 & $1(25.0 \%)$ & $1(8.3)$ \\
\hline Open-angle glaucoma & $8(100.0 \%)$ & $3(75.0 \%)$ & $11(91.7)$ \\
\hline Time since current diagnosis (weeks) & $356.4 \pm 342.39$ & $447.8 \pm 265.05$ & $386.8 \pm 309.49$ \\
\hline \multicolumn{4}{|l|}{ Prior hypotensive therapy } \\
\hline Combination therapy & $1(12.5 \%)$ & $2(50.0 \%)$ & $3(25.0)$ \\
\hline Prostaglandins (monotherapy) & 0 & 0 & 0 \\
\hline Other (monotherapy) & $5(62.5 \%)$ & $2(50.0 \%)$ & $7(58.3)$ \\
\hline No prior therapy & $2(25.0 \%)$ & 0 & $2(16.7)$ \\
\hline Prior prostaglandin therapy & 0 & 0 & 0 \\
\hline
\end{tabular}


Table 2 continued

\begin{tabular}{llll}
\hline $\begin{array}{l}\text { Baseline demographics and clinical } \\
\text { characteristics }\end{array}$ & $\begin{array}{l}\text { Netarsudil treatment arm } \\
(\boldsymbol{n}=\mathbf{8})\end{array}$ & $\begin{array}{l}\text { Vehicle treatment arm } \\
(\boldsymbol{n}=\mathbf{4})\end{array}$ & Total $(\boldsymbol{N}=\mathbf{1 2})$ \\
\hline $\begin{array}{l}\text { Time on current hypotensive therapy } \\
\text { (weeks) }\end{array}$ & $71.3 \pm 141.95$ & $48.3 \pm 50.91$ & $62.1 \pm 110.45$ \\
$\begin{array}{l}\text { IOP study eye }(\mathrm{mmHg}) \\
\text { Screening }\end{array}$ & $18.50 \pm 2.88$ & $17.00 \pm 2.83$ & $18.00 \pm 2.83$ \\
$\quad$ Day 1/day 2, mean nocturnal & $22.84 \pm 2.21$ & $23.56 \pm 1.94$ & $23.08 \pm 2.07$ \\
Day 1/day 2, mean diurnal & $22.03 \pm 2.05$ & $22.31 \pm 0.94$ & $22.13 \pm 1.71$ \\
Day 1/day 2, mean 24 h & $22.44 \pm 2.08$ & $22.94 \pm 1.28$ & $22.60 \pm 1.81$ \\
Central corneal thickness $(\mu \mathrm{m})$ & & & \\
Study eye & $539.63 \pm 30.59$ & $541.63 \pm 31.34$ & $540.29 \pm 29.40$ \\
Fellow eye & $540.63 \pm 30.14$ & $542.13 \pm 27.50$ & $541.13 \pm 28.01$ \\
Cup to disc ratio & & & \\
Study eye & $0.55 \pm 0.076$ & $0.55 \pm 0.058$ & $0.55 \pm 0.067$ \\
Fellow eye & $0.55 \pm 0.076$ & $0.55 \pm 0.058$ & $0.55 \pm 0.067$ \\
Visual field mean deviation $(\mathrm{dB})$ & $-1.40 \pm 3.42$ & & $-0.64 \pm 3.04$ \\
Study eye & $-1.21 \pm 2.66$ & $-0.95 \pm 5.97$ & $-1.12 \pm 3.78$ \\
Fellow eye & & &
\end{tabular}

Values are presented as the mean \pm standard deviation or as a number with/without the percentage in parentheses, unless indicated otherwise

nocturnal IOP was significantly greater in the netarsudil group than in the vehicle group (0.4 mmHg; $P<0.001$ for comparison).

For secondary efficacy endpoints, reduction from baseline in study eye mean diurnal IOP was also statistically significant in the netarsudil group (3.5 mmHg; $P<0.001)$ at day $8 /$ day 9 . (Fig. 2b); this reduction from baseline in mean diurnal IOP was also significantly greater than that observed in the vehicle group $(0.9 \mathrm{mmHg}$; $P<0.01$ for comparison). There was no difference in the reduction from baseline in mean nocturnal IOP $(3.5 \mathrm{mmHg})$ and mean diurnal IOP $(3.5 \mathrm{mmHg})$ with netarsudil.
The reduction from baseline in study eye mean 24-h IOP was statistically significant with netarsudil $(3.5 \mathrm{mmHg} ; P<0.001)$ at day $8 /$ day 9 and significantly greater than the reduction from baseline in mean 24-h IOP with vehicle (0.7 mmHg; $P<0.001$ for comparison). Mean IOP at each time point assessed over $24 \mathrm{~h}$ is shown for netarsudil and vehicle on day $1 /$ day 2 (pre-treatment) and day 8/day 9 (post-treatment) in Fig. 3. Netarsudil significantly reduced IOP versus baseline at each assessed time point (Fig. 3a). Further, the distance between the baseline and post-treatment curves was consistent at each time point, indicating that netarsudil was equally effective at reducing IOP 

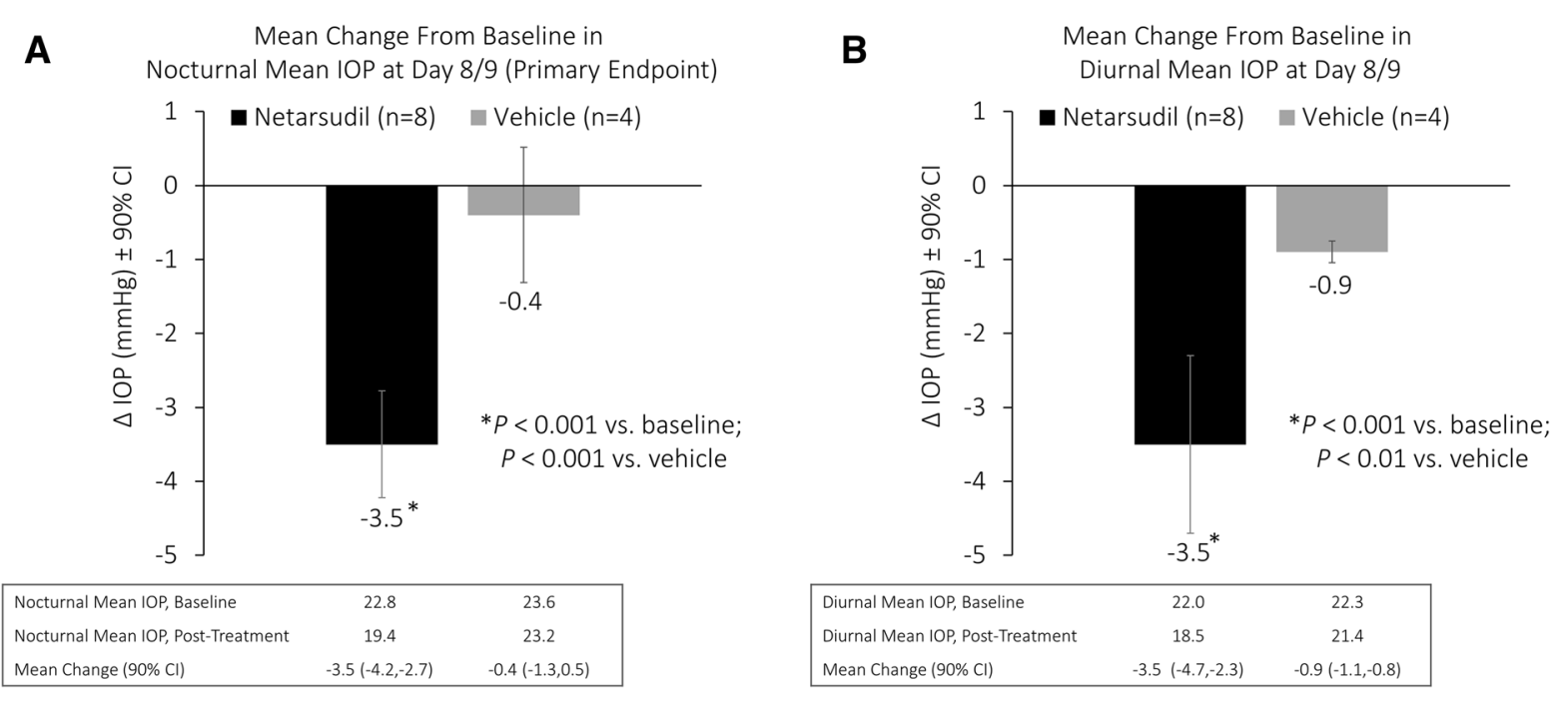

Fig. 2 Change from pre-treatment to post-treatment in study eye mean nocturnal intraocular pressure (a) and mean diurnal intraocular pressure (b). CI Confidence interval
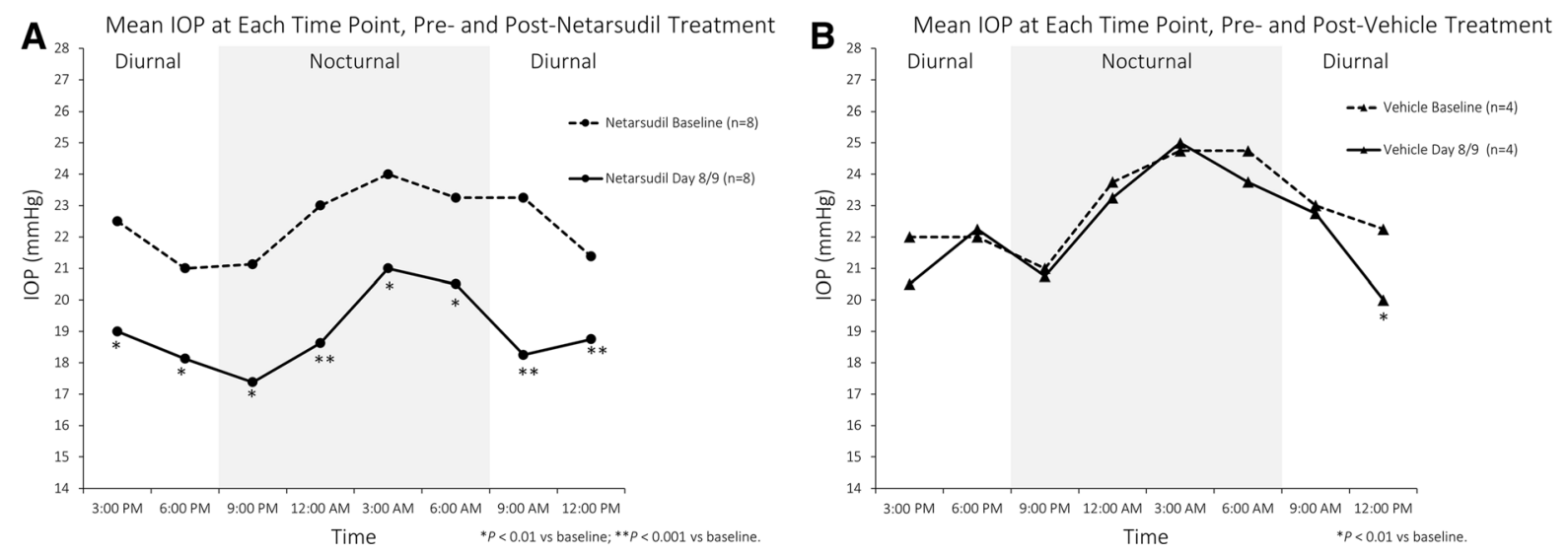

Fig. 3 Study eye mean intraocular pressure at each 24-h time point assessed pre-treatment and post-treatment for netarsudil (a) and vehicle (b)

during both diurnal and nocturnal periods. The vehicle-treated group did not experience significant reduction versus baseline at any time point, with the exception of the 12:00 PM assessment (Fig. 3b).

\section{Safety}

No adverse event of any severity, or any seriousness, related or not to study drug was reported during the study. No subject discontinued from the study due to adverse events.

\section{DISCUSSION}

Netarsudil met the primary efficacy endpoint of this study, demonstrating statistically significant mean reduction from nocturnal mean baseline IOP (3.5 $\mathrm{mmHg}$ ). The nocturnal IOPlowering effect of netarsudil was equivalent to the diurnal effect $(3.5 \mathrm{mmHg})$. The IOP-lowering effect of netarsudil was clinically and statistically significantly greater than vehicle during the nocturnal and diurnal periods.

No tolerability issues were seen in this small study of short duration. Other than the short 
duration of follow-up, another reason for the lack of adverse events may have been the treatment-experienced study population; of the 12 subjects, 10 had been on prior ocular hypotensive medications (other than prostaglandins) for $>1$ year.

The finding that netarsudil maintained consistent IOP reduction throughout a 24 -h period is of considerable interest, given that the IOP reduction obtained at night is less than that obtained in the daytime for several IOP-lowering medication classes. Clinical studies of timolol, brimonidine, dorzolamide, and various prostaglandin analogues (PGAs) that evaluated efficacy over $24 \mathrm{~h}$ have shown either no efficacy or reduced efficacy at night [16-20]. PGAs, which lower IOP primarily by increasing uveoscleral outflow, [21] are the only class of medication that have consistently demonstrated a $>1-\mathrm{mmHg}$ average reduction in IOP during the nocturnal period, although efficacy is still less during the night than during the day $[16,18-20]$. In one study, timolol and dorzolamide decreased nocturnal IOP, although to a lesser extent than latanoprost [16], but subsequent research showed no significant nocturnal reduction in either seated or supine IOP at any time point with either of these agents $[18,22]$. A reason for the lack of nocturnal efficacy may be the mechanisms of action of timolol and dorzolamide, which are mediated through suppression of aqueous humor production. In a recent study, the magnitude of the normal physiologic decrease in aqueous humor production occurring naturally each night (47\%) was nearly twice as large as that achieved by timolol during the daytime [18]. This natural decrease may counter the nighttime efficacy of beta-blockers and carbonic anhydrase inhibitors [23]. The IOP-lowering activity of netarsudil is driven primarily by an increase in trabecular outflow, providing a plausible mechanistic rationale for the consistent 24-h efficacy seen in the present study [22, 24-26].

A methodological strength of this study is the measurement of nocturnal IOP in a habitual (supine) position. Previous observational and interventional studies that evaluated nighttime IOP found sitting values to be lower than supine values, possibly due to the distribution of body fluid in the supine position and increased episcleral venous pressure $[16,18,23]$. Measurement of supine IOP at night is accordingly a conservative methodology. Limitations of the study include the small study population and short duration of follow-up. Additionally, local anesthetic was applied to facilitate IOP measurements with the Perkins tonometer. Topical anesthetic may exert toxic effects on the corneal epithelium, potentially affecting drug penetration into the anterior chamber [27].

\section{CONCLUSIONS}

In this randomized, controlled study, netarsudil ophthalmic solution $0.02 \%$ demonstrated consistent IOP-lowering efficacy over the dosing interval, with equivalent reductions from baseline during the day and at night $(3.5 \mathrm{mmHg})$ and statistically significant reductions versus placebo during both periods. The findings reported here are promising preliminary data on the maintenance of 24-h IOP control with netarsudil and warrant further research.

\section{ACKNOWLEDGEMENTS}

We thank the participants of the study.

Funding Aerie Pharmaceuticals, Inc. (Durham, NC), funded this study. Aerie Pharmaceuticals, Inc. also funded the journal's Rapid Service Fees.

Medical Writing and/or Editorial Assistance Andrew J. Horgan, PhD, of BioScience Communications (New York, NY) provided medical writing and editorial services, activity that was also funded by Aerie Pharmaceuticals, Inc.

Authorship All named authors meet the International Committee of Medical Journal Editors (ICMJE) criteria for authorship for this article, take responsibility for the integrity of the work as a whole, and have given their approval for this version to be published. 
Prior Presentation Data from this study were presented at the 2017 ARVO Annual Meeting, 7-11 May 2017, Baltimore, MD.

Disclosures James H. Peace reports consulting work with Aerie Pharmaceuticals, Alcon, and Allergan. Hayley J. McKee and Casey C. Kopczynski are employees of Aerie Pharmaceuticals, Inc.

Compliance with Ethics Guidelines Institutional ethics approval was obtained from Schulman Institutional Review Board (IRB\# 201604624). The study was conducted in accordance with institutional review board regulations; US Code of Federal Regulations, Title 21; International Conference on Harmonization-Consolidated Good Clinical Practices Guideline (E6); standard operating procedures of the sponsor and any vendors participating in the conduct of the study; and the ethical principles that have their origin in the Declaration of Helsinki. An approved informed consent procedure had to take place before any studyspecific procedures were initiated.

Data Availability Datasets generated during and/or analyzed during the current study are available at https://clinicaltrials.gov/ct2/show/ results/NCT02874846.

Open Access. This article is licensed under a Creative Commons Attribution-NonCommercial 4.0 International License, which permits any non-commercial use, sharing, adaptation, distribution and reproduction in any medium or format, as long as you give appropriate credit to the original author(s) and the source, provide a link to the Creative Commons licence, and indicate if changes were made. The images or other third party material in this article are included in the article's Creative Commons licence, unless indicated otherwise in a credit line to the material. If material is not included in the article's Creative Commons licence and your intended use is not permitted by statutory regulation or exceeds the permitted use, you will need to obtain permission directly from the copyright holder. To view a copy of this licence, visit http://creativecommons.org/licenses/by$\mathrm{nc} / 4.0 /$.

\section{REFERENCES}

1. Tham YC, Li X, Wong TY, Quigley HA, Aung T, Cheng CY. Global prevalence of glaucoma and projections of glaucoma burden through 2040: a systematic review and meta-analysis. Ophthalmology. 2014;121:2081-90.

2. Choi J, Kook MS. Systemic and ocular hemodynamic risk factors in glaucoma. Biomed Res Int. $2015 ; 2015: 141905$.

3. Flaxman SR, Bourne RRA, Resnikoff S, et al. Global causes of blindness and distance vision impairment 1990-2020: a systematic review and meta-analysis. Lancet Glob Health. 2017;5:e1221-34.

4. AGIS Investigators. The Advanced Glaucoma Intervention Study (AGIS): 7. The relationship between control of intraocular pressure and visual field deterioration. Am J Ophthalmol. 2000;130:429-40.

5. Heijl A, Leske MC, Hyman L, et al. Intraocular pressure reduction with a fixed treatment protocol in the early manifest glaucoma trial. Acta Ophthalmol. 2011;89:749-54.

6. Kass MA, Heuer DK, Higginbotham EJ, et al. The ocular hypertension treatment study. Arch Ophthalmol. 2002;120:701-13.

7. Kass MA, Gordon MO, Gao F, et al. Delaying treatment of ocular hypertension: the ocular hypertension treatment study. Arch Ophthalmol. 2010;128: 276-87.

8. Collaborative Normal-Tension Glaucoma Study Group. The effectiveness of intraocular pressure reduction in the treatment of normal-tension glaucoma. Am J Ophthalmol. 1998;126:498-505.

9. Anderson DR. Collaborative normal tension glaucoma study. Curr Opin Ophthalmol. 2003;14: 86-90.

10. Asrani S, Zeimer R, Wilensky J, et al. Large diurnal fluctuations in intraocular pressure are an independent risk factor in patients with glaucoma. J Glaucoma. 2000;9:134-42.

11. Bagga H, Liu JH, Weinreb RN. Intraocular pressure measurements throughout the $24 \mathrm{~h}$. Curr Opin Ophthalmol. 2009;20:79-83. 
12. Costa VP, Harris A, Anderson D, et al. Ocular perfusion pressure in glaucoma. Acta Ophthalmol. 2014;92:e252-66.

13. Choi J, Jeong J, Cho HS, Kook MS. Effect of nocturnal blood pressure reduction on circadian fluctuation of mean ocular perfusion pressure: a risk factor for normal tension glaucoma. Invest Ophthalmol Vis Sci. 2006;47:831-6.

14. Sung KR, Lee S, Park SB, et al. Twenty-four hour ocular perfusion pressure fluctuation and risk of normal-tension glaucoma progression. Invest Ophthalmol Vis Sci. 2009;50:5266-74.

15. Serle JB, Katz LJ, McLaurin E, ROCKET-1 and ROCKET-2 Study Groups, et al. Two phase 3 clinical trials comparing the safety and efficacy of netarsudil to timolol in patients with elevated intraocular pressure: Rho Kinase Elevated IOP Treatment Trial 1 and 2 (ROCKET-1 and ROCKET-2). Am J Ophthalmol. 2018;186:116-27.

16. Orzalesi N, Rossetti L, Invernizzi $\mathrm{T}$, Bottoli $\mathrm{A}$, Autelitano A. Effect of timolol, latanoprost, and dorzolamide on circadian IOP in glaucoma or ocular hypertension. Invest Ophthalmol Vis Sci. 2000;41:2566-73.

17. Liu JH, Medeiros FA, Slight JR, et al. Diurnal and nocturnal effects of brimonidine monotherapy on intraocular pressure. Ophthalmology. 2010;117: 2075-9.

18. Gulati V, Fan S, Zhao M, Maslonka MA, Gangahar $\mathrm{C}$, Toris CB. Diurnal and nocturnal variations in aqueous humor dynamics of patients with ocular hypertension undergoing medical therapy. Arch Ophthalmol. 2012;130:677-84.

19. Tung JD, Tafreshi A, Weinreb RN, Slight JR, Medeiros FA, Liu JH. Twenty-four-hour effects of bimatoprost $0.01 \%$ monotherapy on intraocular pressure and ocular perfusion pressure. BMJ Open. 2012;2:e001106.

20. Seibold LK, Kahook MY. The diurnal and nocturnal effect of travoprost with sofZia on intraocular pressure and ocular perfusion pressure. Am J Ophthalmol. 2014;157:44-49.e1.

21. Toris CB, Gabelt BT, Kaufman PL. Update on the mechanism of action of topical prostaglandins for intraocular pressure reduction. Surv Ophthalmol. 2008;53(Suppl 1):S107-20.

22. Li G, Mukherjee D, Navarro I, et al. Visualization of conventional outflow tissue responses to netarsudil in living mouse eyes. Eur J Pharmacol. 2016;787: 20-31.

23. Fan S, Hejkal JJ, Gulati V, Galata S, Camras CB, Toris $\mathrm{CB}$. Aqueous humor dynamics during the day and night in volunteers with ocular hypertension. Arch Ophthalmol. 2011;129:1162-6.

24. Wang RF, Williamson JE, Kopczynski C, Serle JB. Effect of $0.04 \%$ AR-13324, a ROCK, and norepinephrine transporter inhibitor, on aqueous humor dynamics in normotensive monkey eyes. J Glaucoma. 2015;24:51-4.

25. Ren R, Li G, Le TD, Kopczynski C, Stamer WD, Gong $H$. Netarsudil increases outflow facility in human eyes through multiple mechanisms. Invest Ophthalmol Vis Sci. 2016;57:6197-209.

26. Kazemi A, McLaren JW, Kopczynski CC, Heah TG, Novack GD, Sit AJ. The effects of netarsudil ophthalmic solution on aqueous humor dynamics in a randomized study in humans. J Ocul Pharmacol Ther. 2018;34(5):380-6.

27. Patel M, Fraunfelder FW. Toxicity of topical ophthalmic anesthetics. Expert Opin Drug Metab Toxicol. 2013;9(8):983-8. 